\title{
MANY FACES OF LATTICE TOLERANCES
}

\begin{abstract}
Our aim is to overview and discuss some of the most popular approaches to the notion of a tolerance relation in algebraic structures with the special emphasis on lattices.
\end{abstract}

Keywords: lattice, tolerance, congruence, covering system, gluing.

\section{Introduction}

The idea of tolerance relations seen as a formalization of the intuitive notion of resemblance was present in the late works of Poincaré who introduced the sets of impressions to describe sensations concerning objects hardly indiscernible [2].

In 1962 Zeeman formally introduced the notion of a tolerance as a relation that is reflexive and symmetric, but not necessarily transitive [29]. Studying models of visual perceptions, Zeeman found it useful to axiomatize the notion of similarity and formalized the notion of tolerance spaces. The idea of "being within tolerance" or of "closeness" or "resemblance" is universal enough to appear, quite naturally, in almost any setting. It is particularly natural in practical applications: real-life problems, more often than not, deal with approximate input data and require only viable results with a tolerable level of exactness. Therefore, the topic became popular among researchers from different areas such as linguistics, information theory, humanities, social sciences, but also logic and mathematics. Studies on tolerance spaces were conducted by Szrejder [28], Arbib [1], Pogonowski [27] and others.Since then, numerous books and papers concerning this topic have appeared. 
There are different approaches to tolerance relations, so we decided to present here a short survey of them. This paper is meant as an inspiration to wider and more complete studies of results obtained in this area, particularly in algebraic structures.

As a natural generalization of congruences, tolerances appeared to be a very useful tool in universal algebra. In an algebraic structure $\mathcal{A}=$ $(A, F)$ by tolerances we mean only those reflexive and symmetric relations which are compatible with the operations from $F$. Some authors (see, e.g., [23]) call them admissible relations. Formally speaking, a reflexive and symmetric relation $R$ on an algebra $\mathcal{A}=(A, F)$ is a tolerance iff for every $n$-ary operation $f \in F$ and for all $a_{1}, \ldots, a_{n}, b_{1}, \ldots, b_{n} \in A$, such that $\left(a_{i}, b_{i}\right) \in T$ for $i=1, \ldots, n$ we have $\left(f\left(a_{1}, \ldots, a_{n}\right), f\left(b_{1}, \ldots, b_{n}\right)\right) \in T$.

Therefore, $R \subseteq A^{2}$ is a tolerance on an algebra $\mathcal{A}=(A, F)$ iff $R$ is reflexive, symmetric and closed under the operations from $F$ (according to coordinates). In other words, it means that $R$ is a reflexive and symmetric subalgebra of $\mathcal{A}^{2}$.

It is clear that every congruence of an algebra $\mathcal{A}$ is a tolerance on it and then the notion of a tolerance in universal algebra can be regarded as a generalization of the notion of a congruence.

Tolerances play an important role in the theory of Maltsev conditions (see for example [25] or [12]). Moreover, as we show in the next sections, they are particularly useful in lattice theory.

There are many ways to deal with tolerances, the most common ones consist in describing tolerances as some types of covering systems (e.g., $[4,19])$ or characterizing them as homomorphic images of congruences (see [11]). In the case of finite lattices we can also use polarities [23] or Galois connections [17].

\section{Covering systems}

The most natural way of studying a tolerance is by considering the system of its blocks.

Let $T$ be a tolerance on a given algebra $\mathcal{A}=(A, F)$ (or even more general, on a given set $A$ ). Since tolerances are a generalization of congruences (equivalence relations, respectively) and every congruence determines uniquely a quotient algebra (a partition of a given set), we should look for a generalization of the concept of a congruence class (or an equivalence class, in general). 
In fact, there are two possible ways of performing this task. First, we can consider a class $[a]_{T} \subseteq A$ containing a given element $a \in A$ defined as the set of all elements from $A$ tolerant with $a$ :

$$
[a]_{T}=\{b \in A:(a, b) \in T\} .
$$

On the other hand, we can consider subsets $B \subseteq A$ such that $B^{2} \subseteq T$. Such subsets are called preblocks of $T$. Blocks of $T$ are maximal (under inclusion) preblocks of $T$.

It is easy to check that in the case of equivalence relations both the above definitions coincide and lead to the same notion of equivalence classes. However, in general, it is not true for tolerance relations.

EXAMPLE 1. Let us consider the set of cookies of two sizes: big and small, each of them being either red or yellow. We say that two cookies are similar if they are both of the same size or colour. It is obvious that this relation of similarity among cookies is a tolerance. Let us observe that the tolerance class containing a small red cookie consists of all cookies that are small or red. At the same time there are two different blocks containing this cookie: the block of red cookies and the block of small cookies. Of course, they are not disjoint and their common part consists of small red cookies.

EXAMPLE 2. Let us consider the lattice $\mathcal{L}$ shown in Figure 1 and the tolerance $T$ generated on it by all pairs $(x, y) \in L^{2}$ such that $x \prec y$.

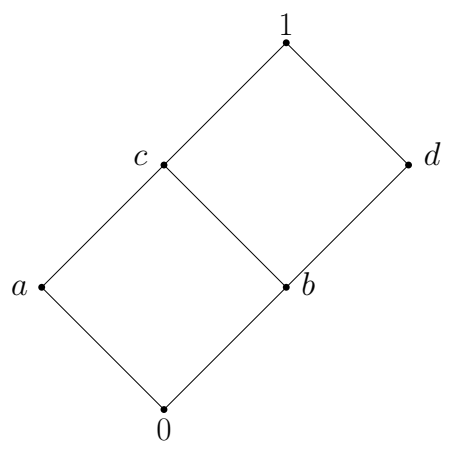

Fig. 1. Lattice $\mathcal{L}$ from Example 2

Since $(0, a),(0, b) \in T$, by compatibility of $T$ with lattice operations, we get $(0, c)=(0 \vee 0, a \vee b) \in T$. Similarly, we obtain $(b, 1) \in T$. However, 
it is not true that $(0, d) \in T$. Therefore, there are two different blocks of $T$ containing $b:\{0, a, b, c\}$ and $\{b, c, d, 1\}$ but $[b]_{T}=L$.

In general, neither classes nor blocks of a tolerance are disjunctive. The fact that they can overlap constitutes an essential difference between congruences and tolerances, which makes the second ones useful in many practical applications, for example in the rough set theory (see, e.g., [26] or [24]) or in the conceptual analysis (see [17]).

In fact, as it follows from the result below, only the notion of blocks is significant.

Lemma 3. Let $T$ be a tolerance relation on a set $A$ and let $A / T$ denote the set of all blocks of $T$ on $A$. Then, for every $a \in A$, we have

(i) $[a]_{T}=\bigcup\{\alpha \in A / T: a \in \alpha\}$;

(ii) $\alpha \in A / T$ iff $\alpha=\bigcap\left\{[b]_{T}: b \in \alpha\right\}$.

A proof is identical as in the case of algebraic tolerances, which can be found in [4]. If we deal with tolerances on an algebraic structure, blocks can be characterized by means of an algebraic function over the given structure ([4]).

There are many papers investigating blocks of tolerances of different algebras. In particular, Chajda and Duda proved in [7] what follows.

TheOrem 4. Let $T$ be a tolerance on an algebra $\mathcal{A}$. Every block of $T$ is a subalgebra of $\mathcal{A}$ iff $\mathcal{A}$ is idempotent.

Let us recall that an algebra $\mathcal{A}=(A, F)$ is idempotent iff its every operation is idempotent, i.e., $f(a, \ldots, a)=a$ for every $f \in F$ and $a \in A$.

Since every lattice is an idempotent algebra, we conclude immediately that blocks of tolerances of a lattice are its sublattices. What is more, they are always convex sublattices (see [9] or [3]).

It is well-known that there is a one-to-one correspondence between congruences of a given algebra $\mathcal{A}=(A, F)$ and these partitions of the underlying set $A$ which preserve the substitution property for all operations from $F$. As it was proved independently by Grätzer and Wenzel in [18] and by Chajda, Niederle and Zelinka in [8], there is a similar one-to-one correspondence between tolerance relations of an algebra $\mathcal{A}=(A, F)$ and a normal covering system of subsets of the set $A$.

The covering system $\left\{\alpha_{i}\right\}_{i \in I}$ of the set $A$ is called normal if it fulfills the following conditions: 
(i) it forms an antichain;

(ii) it fulfills the substitution property for the set $F$ of operations of the algebra $\mathcal{A}$, i.e., for every $n$-ary operation $f \in F$ and all $i_{1}, \ldots, i_{n} \in I$ there exists $j \in I$ such that $f\left(\alpha_{i_{1}}, \ldots, \alpha_{i_{n}}\right) \subseteq \alpha_{j}$;

(iii) for every $B \subseteq A$, if $B \nsubseteq \alpha_{i}$ for every $i \in I$, then there exists $C \subseteq B$ such that $C$ contains exactly two elements and $C \nsubseteq \alpha_{i}$ for every $i \in I$.

All classes of a given congruence of an algebra $\mathcal{A}=(A, F)$ form an algebra (called the quotient algebra) which belongs to the variety generated by $\mathcal{A}$. In the case of tolerances the situation is different. It is not difficult (see, e.g., [4]) to find examples of an algebra $\mathcal{A}$ and a tolerance $T$ on it such that the basic operations from $\mathcal{A}$ cannot be defined uniquely on the blocks of $T$.

EXAMPLE 5. Let us consider an algebra $\mathcal{A}=(\{a, b, c\}, f)$ with a binary operation defined by $f(x, y)=a$ for all $x, y \in\{a, b, c\}$. Let $T$ be a tolerance on $\mathcal{A}$ generated by the set $\{(a, b),(a, c)\}$. Since $(b, c) \notin T$, there are two blocks of $T: \alpha=\{a, b\}$ and $\beta=\{a, c\}$. Notice that

$$
f(\alpha, \beta)=\{f(x, y): x \in \alpha, y \in \beta\}=\{b\} .
$$

Thus, we cannot define uniquely the result of operation $f$ on blocks $\alpha$ and $\beta$, as $\{b\}$ is a subset both $\alpha$ and $\beta$.

Even if it is possible to define the operations on blocks of a tolerance $T$ of an algebra $\mathcal{A}$ uniquely, the corresponding quotient algebra need not belong to the variety generated by $\mathcal{A}$, as we can see later in Example 4 .

Formally speaking, an algebra $\mathcal{A}=(A, F)$ is called tolerance factorable if for every tolerance $T$ on $\mathcal{A}$, every $f \in F$ and every blocks $\alpha_{1}, \ldots, \alpha_{n}$ of $T$ there is a unique block $\beta$ of $T$ such that

$$
\left\{f\left(a_{1}, \ldots, a_{n}\right): a_{1} \in \alpha_{1}, \ldots, a_{n} \in \alpha_{n}\right\} \subseteq \beta .
$$

If $\mathcal{A}$ is tolerance factorable, then for every tolerance $T$ on $\mathcal{A}$ it is possible to form the quotient algebra $\mathcal{A} / T$ by defining $\beta:=f\left(\alpha_{1}, \ldots, \alpha_{n}\right)$ for every $n$-ary operation $f \in F$ and every system of blocks $\alpha_{1}, \ldots, \alpha_{n}$ of $T$. The quotient algebra in the case of tolerances not being congruences is called the factor algebra.

We say that a variety $\mathcal{V}$ is tolerance factorable if all its algebras are tolerance factorable. A variety $\mathcal{V}$ is called strongly tolerance factorable if it is tolerance factorable and for every $\mathcal{A} \in \mathcal{V}$ and every tolerance $T$ on $\mathcal{A}$ it holds $\mathcal{A} / T \in \mathcal{V}$. 
It is clear that every tolerance-trivial algebra, i.e., algebra without tolerances not being congruences, is tolerance factorable. Therefore, for example the variety of Boolean algebras is not only tolerance factorable but also strongly tolerance factorable. The essential question remains, which algebras with proper tolerances enjoy that property.

In 1982 Czédli [10] proved that the variety of lattices is strongly tolerance factorable, although it is not tolerance-trivial. Up to 2012 no other example of such a variety was known. In [5] Chajda, Czédli and Halaš proved that this property is preserved by forming independent joins of varieties, providing infinitely many strongly tolerance factorable varieties with proper tolerances.

Anyway, it is the variety of lattices which behaves surprisingly well with respect to tolerance relations. The fact was discovered and investigated by Czédli, who proved in [10] that if $\mathcal{L}$ is a lattice and $T$ is a tolerance on $\mathcal{L}$, then for any blocks $\alpha, \beta$ of $T$ there is exactly one block containing $\alpha \wedge \beta=\{a \wedge b: a \in \alpha, b \in \beta\}$ and exactly one block containing $\alpha \vee \beta=$ $\{a \vee b: a \in \alpha, b \in \beta\}$, which means that we can form a quotient structure $\mathcal{L} / T$. Moreover, the structure is a lattice and the partial order of blocks in it coincides with the order of ideals generated by them in the lattice of ideals of $\mathcal{L}$ and - by the duality principle - it is dual to the order of filters generated by the blocks in the lattice of filters of $\mathcal{L}$. Formally, if we denote by $(A]$ and $[A)$, respectively, an ideal and a filter generated by the subset $A$ of a lattice $\mathcal{L}$, then for any $\alpha, \beta \in L / T$,

$$
\alpha \leq \beta \text { iff }(\alpha] \subseteq(\beta] \text { iff }[\beta) \subseteq[\alpha) .
$$

However, even in the case of lattices, many properties typical for quotient structures are not, in general, valid for factor structures. Some of them, like the homomorphism theorem and the second isomorphism theorem for lattice congruences, can be imitated in the set of all tolerances partially ordered by a particular restriction of a regular (i.e., inclusion) order of tolerances (see [20]). The resulted poset is not always a lattice, but it can be converted into a specific commutative join-directoid.

In [10], Czédli formulated also a set of necessary and sufficient conditions for a covering of the underlying set $L$ of a lattice $\mathcal{L}$ to be its normal covering.

Theorem 6. Let $\mathcal{L}=(L, \wedge, \vee)$ be a lattice. The covering $\left\{\alpha_{i}\right\}_{i \in I}$ of $L$ is the family of blocks of a certain tolerance $T$ on $\mathcal{L}$ iff the following conditions hold: 
1. Every $\alpha_{i}$ for $i \in I$ is a sublattice of $\mathcal{L}$.

2. For every $i, j \in I$

$$
\left[\alpha_{i}\right)=\left[\alpha_{j}\right) \text { iff }\left(\alpha_{i}\right]=\left(\alpha_{j}\right] .
$$

3. For every $i, j \in I$ there exist $m, n \in I$ such that

$$
\begin{aligned}
& \left\{a \vee b: a \in\left[\alpha_{i}\right), b \in\left[\alpha_{j}\right)\right\}=\left[\alpha_{m}\right), \\
& \left\{a \vee b: a \in\left(\alpha_{i}\right], b \in\left(\alpha_{j}\right]\right\} \subseteq\left(\alpha_{m}\right], \\
& \left\{a \wedge b: a \in\left[\alpha_{i}\right), b \in\left[\alpha_{j}\right)\right\} \supseteq\left[\alpha_{n}\right), \\
& \left\{a \wedge b: a \in\left(\alpha_{i}\right], b \in\left(\alpha_{j}\right]\right\}=\left(\alpha_{n}\right] .
\end{aligned}
$$

4. Let $x \in L, b \in \alpha_{i}$ for some $i \in I$. If for any $b \in \alpha_{i} \cap(a]$ there is $j \in J$ such that $\{b, x\} \subseteq \alpha_{j}$, then $x \in\left(\alpha_{j}\right]$. Dually, if for any $b \in \alpha_{i} \cap[a)$ there is $j \in J$ such that $\{b, x\} \subseteq \alpha_{j}$, then $x \in\left[\alpha_{j}\right)$.

5. If for any convex sublattice $\mathcal{K}$ of $\mathcal{L}$ and any $a, b \in K$ there is $i \in I$ such that $a, b \in \alpha_{i}$, then $K \subseteq \alpha_{j}$ for some $j \in I$.

Moreover, if $\mathcal{L}$ is a finite lattice, then the last two conditions follow from the conditions $1-3$.

In the case of lattices of finite height, blocks of any tolerance relation as convex sublattices - are intervals and hence as the corollary of the above theorem we obtain the following theorem (see [18]).

Theorem 7. Let $\mathcal{L}=(L, \wedge, \vee)$ be a lattice of a finite height. The covering $\left\{\alpha_{i}\right\}_{i \in I}$ of $L$ is the family of blocks of a certain tolerance $T$ on $\mathcal{L}$ iff the following conditions hold:

1. Every $\alpha_{i}$ for $i \in I$ is of the form $\left[0_{i}, 1_{i}\right]$, where $0_{i}, 1_{i} \in L$ and $0_{i}<1_{i}$.

2. For distinct $i, j \in I, 0_{i} \neq 0_{j}$ and $1_{i} \neq 1_{j}$.

3. For any $i, j \in I$ there are $m, n \in I$ such that

$$
\begin{aligned}
& 1_{i} \vee 1_{j} \leq 1_{m}, \quad 1_{i} \wedge 1_{j}=1_{n}, \\
& 0_{i} \vee 0_{j}=0_{m}, \quad 0_{i} \wedge 0_{j} \geq 0_{n} .
\end{aligned}
$$

\section{Gluings and polarities}

As we see from the previous section, any tolerance on a finite lattice (or more general, on a lattice of a finite height) decomposes the lattice into intervals, which themselves form a lattice (the quotient lattice). The intervals can be seen as the partial maps in an atlas and the factor lattice can 
be regarded as a general map indicating the relations between the partial maps. This attitude was adopted particularly by Wille in the theory of concept lattices, which forms a part of the theoretical basis for investigations on artificial intelligence ([17]).

The situation simplifies further when we focus on glued tolerances of a lattice $\mathcal{L}$. A tolerance $T$ on $\mathcal{L}$ is called glued if its transitive closure is the total relation on the lattice.

In [13], Herrmann and Day proved that every finite lattice can be seen as an $\mathcal{L} / T$-gluing of a family of blocks of any glued tolerance $T$ on $\mathcal{L}$. The notion of $\mathcal{K}$-gluing, where $\mathcal{K}$ is a finite lattice, was introduced by Herrmann in [21] as a natural generalization of the original Hall-Dilworth construction of gluing a filter and an ideal ([15]).

Wille describes the idea of Herrmann's construction as analogous to that used in drawing maps. If the area supposed to be set out in a map is too big to make the map legible, we can split it up into an atlas - a collection of maps covering the area together with an additional map which provides the information how the partial maps are related. It is common that the partial maps overlap to make data more accessible. Of course, the partial maps can be "glued" together to obtain the map of the whole area.

Let $\left(\mathcal{L}_{i}\right)_{i \in K}$ be a family of finite lattices and let an index set $K$ be the underlying set of a finite lattice $\mathcal{K}=(K, \leq)$. The family $\left(\mathcal{L}_{i}\right)_{i \in K}$ is called a $\mathcal{K}$-atlas with overlapping neighbours if the following conditions hold for all $i, j \in K$ :

(i) if $L_{i} \subseteq L_{j}$, then $i=j$;

(ii) if $i<j$ and there is no $k \in K$ such that $i<k<j$, then $L_{i} \cap L_{j} \neq \emptyset$;

(iii) if $i<j$ and $L_{i} \cap L_{j} \neq \emptyset$, then the orders of lattices $\mathcal{L}_{i}$ and $\mathcal{L}_{j}$ coincide on $L_{i} \cap L_{j}$ and the interval $L_{i} \cap L_{j}$ is at the same time a filter of $\mathcal{L}_{i}$ and an ideal of $\mathcal{L}_{j}$;

(iv) $L_{i} \cap L_{j}=L_{i \wedge j} \cap L_{i \vee j}$.

The structure $\mathcal{L}=\left(\bigcup L_{i \in K}, \leq\right)$, where $\leq$ is the transitive closure of the union of orders of all lattices $\mathcal{L}_{i}$ for $i \in K$, is called the sum of the $\mathcal{K}$-atlas with overlapping neighbours or simply the $\mathcal{K}$-gluing of the family $\left(\mathcal{L}_{i}\right)_{i \in K}$.

One can prove the following (see [13] or [17]).

THEOREM 8. Let $\left(\mathcal{L}_{i}\right)_{i \in K}$ be a $\mathcal{K}$-atlas with overlapping neighbours. The sum of the $\mathcal{K}$-atlas is a lattice $\mathcal{L}$ for which the lattices $\mathcal{L}_{i}$, where $i \in K$, are blocks of some glued tolerance $T$ on $\mathcal{L}$ and the mapping $i \mapsto L_{i}$ is an isomorphism of $\mathcal{K}$ onto the factor lattice $\mathcal{L} / T$. 
Conversely, if $T$ is a glued tolerance on a lattice $\mathcal{L}$, then the family of blocks of $T$ forms an $\mathcal{L} / T$-atlas with overlapping neighbours, whose $\mathcal{L} / T$ gluing is the lattice $\mathcal{L}$.

Since, as it is easy to notice, the intersection of a family of glued tolerances on a lattice $\mathcal{L}$ is again a glued tolerance, then there is the smallest (under inclusion) glued tolerance, which is called the skeleton tolerance of $\mathcal{L}$. The factor lattice of $\mathcal{L}$ by the skeleton tolerance is said to be the skeleton $S(\mathcal{L})$ of $\mathcal{L}$. Therefore, any finite lattice $\mathcal{L}$ can be seen as the $S(\mathcal{L})$-gluing of the blocks of its skeleton. It is particularly useful in the case of finite modular or distributive lattices since the blocks of their skeleton tolerance are regular and easy to describe (see for example $[22,14,19]$ ).

Example 9 . Figure 3 depicts a $\mathcal{K}$-atlas with overlapping neighbours along with the lattice $\mathcal{K}$. The $\mathcal{K}$-gluing of this atlas gives the three generated free distributive lattice $\mathcal{F}_{D}(3)$ presented in Figure 2. On the other hand, $\mathcal{B}_{i}$, where $i \in K$, are the blocks of the skeleton tolerance of $\mathcal{F}_{D}(3)$ and $\mathcal{K}=S\left(\mathcal{F}_{D}(3)\right)$.

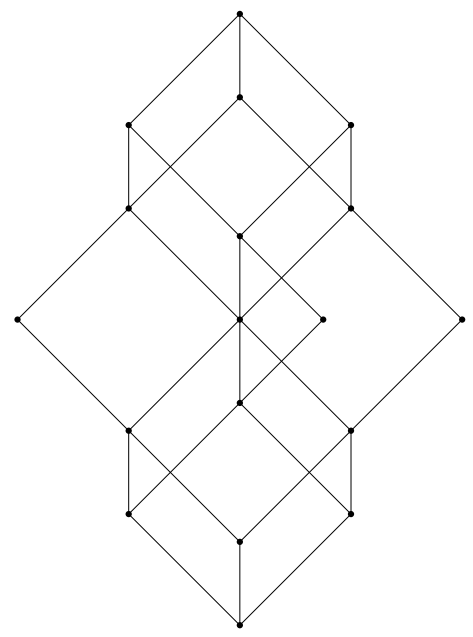

Fig. 2. The lattice $\mathcal{F}_{D}(3)$

We can notice now that the factor lattice of a distributive lattice need not be distributive, so it need not belong to the variety generated by it. 

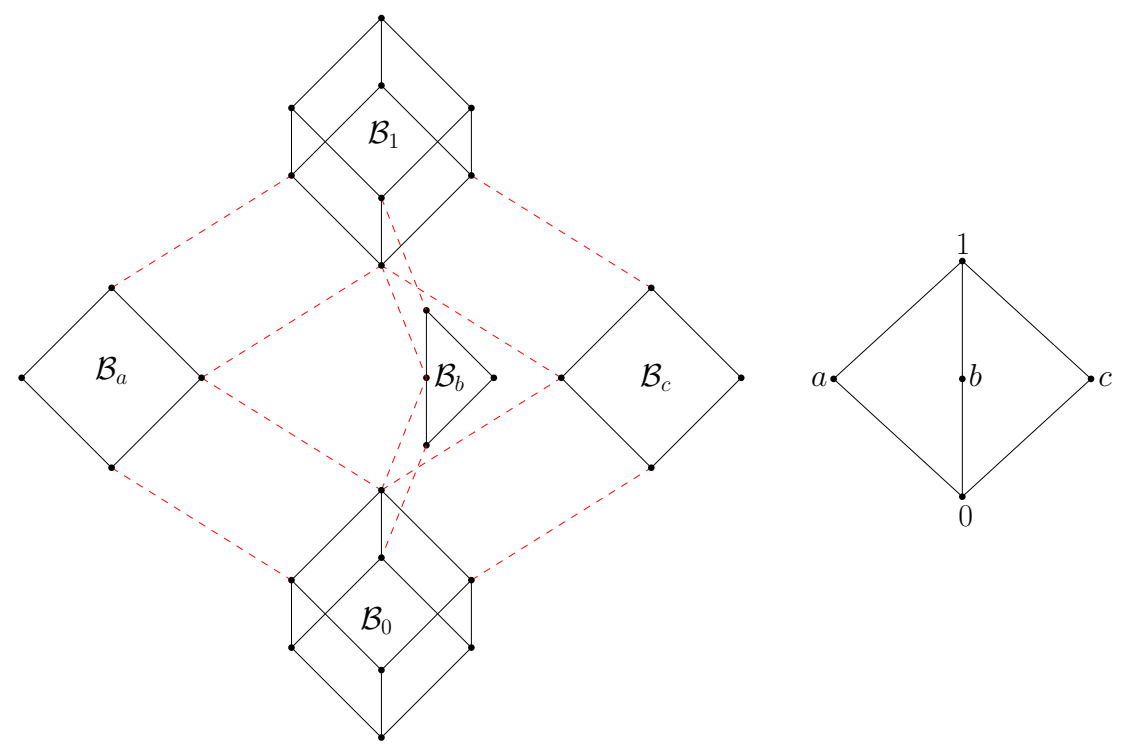

Fig. 3. The $\mathcal{K}$-atlas with overlapping neighbours (left) and the lattice $\mathcal{K}$ (right)

There is also another way of describing tolerances on finite lattices, namely by means of polarities (see [23]).

By a polarity in a lattice $\mathcal{L}$ we mean a pair $(f, g)$ of mappings $L \rightarrow L$ such that $f$ is a decreasing $\vee$-endomorphism, $g$ is an increasing $\wedge$-endomorphism and $f(g(x)) \leq x \leq g(f(x))$ for every $x \in L$.

Hobby and McKenzie observed that there is a one-to-one connection between polarities and tolerance relations in finite lattices.

THEOREM 10. Let $\mathcal{L}$ be a finite lattice. If $T$ is a tolerance on $\mathcal{L}$, then

$$
\begin{aligned}
f(x) & :=\bigwedge\{y:(x, y) \in T\}, \\
g(x) & :=\bigvee\{y:(x, y) \in T\}
\end{aligned}
$$

define the polarity $(f, g)$ such that

$$
T=\{(x, y): f(x \vee y) \leq x \wedge y\} .
$$

On the other hand, if $(f, g)$ is a polarity in a lattice $\mathcal{L}$, then there is exactly one tolerance on $\mathcal{L}$ such that $f, g, T$ fulfill the above conditions. 
Additionally, the tolerance $T$ is glued iff the $\wedge$-endomorphism $g$ defined above is strictly decreasing (or, what is equivalent, the $\vee$-endomorphism $f$ is strictly increasing).

\section{Homomorphic images of congruences}

Let $\mathcal{A}=(A, F)$ and $\mathcal{B}=\left(B, F^{\prime}\right)$ be algebras with the same signature. If $\phi: \mathcal{A} \rightarrow \mathcal{B}$ is a surjective homomorphism and $T$ is a tolerance on $\mathcal{A}$, then $\phi(T)=\{(\phi(x), \phi(y)):(x, y) \in T\}$ is a tolerance on $\mathcal{B}$ (see [16]). In particular, if $T$ is congruence, then $\phi(T)$ is a tolerance, but not necessarily a congruence. In other words, the homomorphic image of any congruence is a tolerance.

Czédli and Grätzer proved in [11] that in the case of lattices the inverse holds, i.e., every lattice tolerance is a homomorphic image of some lattice congruence.

TheOREM 11. Let $T$ be a tolerance of a lattice $\mathcal{L}$. Then there are a lattice $\mathcal{K}$, a congruence $\theta$ on $\mathcal{K}$ and a surjective homomorphism $\phi: \mathcal{K} \rightarrow \mathcal{L}$ such that $T=\phi(\theta)$.

The construction of the lattice $\mathcal{K}$, the congruence $\theta$ and the homomorphism $\phi$ is very natural and based on facts proved by Czédli in [10]. Namely,

$$
K:=\{(\alpha, a): \alpha \in L / T, a \in \alpha\},
$$

the lattice operations on $K$ are defined coordinate-wise, i.e.,

$$
(\alpha, a) \wedge(\beta, b):=(\alpha \wedge \beta, a \wedge b),
$$

and dually for $\vee$. The congruence $\theta$ is defined by

$$
((\alpha, a),(\beta, b)) \in \theta \text { iff } \alpha=\beta
$$

and the homomorphism is given by $(\alpha, a) \mapsto a$.

Czédli and Kiss in [12] characterized by means of a Maltsev-like condition those varieties in which tolerances are homomorphic images of congruences of some algebras within the variety. They observed that among them there are all varieties of lattices, all varieties of unary algebras and the variety of semilattices. Chajda, Czédli, Halas and Lipparini proved in [6] that also all varieties defined by a set of linear equations enjoy that property. By a linear identity they mean an identity $s=t$ such that each variable occurs at most once in each of the terms $s$ and $t$. 


\section{References}

[1] M. Arbib, Tolerance automata, Kybernetika, Vol. 3 (1967), pp. 223-233.

[2] H. Poincaré (Author) and J. W. Bolduc (Translator), Mathematics and Science: Last essays (1913), Kessinger Publishing, 2010.

[3] H. J. Bandelt, Tolerance relations of lattices, Bulletin of the Australian Mathematical Society, Vol. 23 (1981), pp. 367-381.

[4] I. Chajda, Algebraic Theory of Tolerance Relations, Univerzita Palackého Olomouc, Olomouc, 1991.

[5] I. Chajda, G. Czédli, and R. Halaš, Independent joins of tolerance factorable varieties, Algebra Universalis, Vol. 69 (2013), pp. 83-92.

[6] I. Chajda, G. Czédli, R. Halaš, and P. Lipparini, Tolerances as images of congruences in varieties defined by linear identities, Algebra Universalis, Vol. 69 (2013), pp. 167-169.

[7] I. Chajda and J. Duda, Blocks of binary relations, Annales Universitatis Scientiarium Budapestinensis, Sectio Mathematica, Vol. 13-14 (1979-1980), pp. 3-9.

[8] I. Chajda, J. Niederle, and B. Zelinka, On existence conditions for compatible tolerances, Czechoslovak Mathematical Journal, Vol. 2, (1976), pp. 304-311.

[9] I. Chajda and B. Zelinka, Lattices of tolerances, Casopis pro pestovani matematiky, Vol. 102 (1977), pp. 10-24.

[10] G. Czédli, Factor lattices by tolerances, Acta Scientiarum Mathematicarum (Szeged), Vol. 44 (1982), pp. 35-42.

[11] G. Czédli and G. Grätzer, Lattice tolerances and congruences, Algebra Universalis, Vol. 66 (2011), pp. 5-6.

[12] G. Czédli and E. W. Kiss, Varieties whose tolerances are homomorphic images of their congruences, Bulletin of the Australian Mathematical Society, Vol. 87 (2013), pp. 326-338.

[13] A. Day and Ch. Herrmann, Gluings of modular lattices, Order, Vol. 5 (1988), pp. 85-101.

[14] A. Day and B. Jónsson, Non-arguesian configurations and gluings of modular lattices, Algebra Universalis, Vol. 26 (1989), pp. 208-215. 
[15] R. P. Dilworth, Lattices with unique irreducible decompositions, Annals of Mathematics, Vol. 41, No. 2 (1940), pp. 771-777.

[16] E. Fried and G. Grätzer, Notes on tolerance relations of lattices: A conjecture of R.N. McKenzie, Journal of Pure and Applied Algebra, Vol. 68 (1990), pp. 127-134.

[17] B. Ganter and R. Wille, Formal concept analysis. Mathematical Foundations, Springer-Verlag, 1999.

[18] G. Grätzer and G. H. Wenzel, Notes on tolerance relations on lattices, Acta Scientiarum Mathematicarum (Szeged), Vol. 54 (1990), pp. 229-240.

[19] J. Grygiel, The concept of gluing for lattices, Wydawnictwo WSP Częstochowa, 2004.

[20] J. Grygiel and S. Radeleczki, On the tolerance lattice of tolerance factors, Acta Mathematica Hungarica, Vol. 141, No. 3 (2013), pp. 220-237.

[21] Ch. Herrmann, S-verklebte Summen von Verbänden, Mathematische Zeitschrift, Vol. 130 (1973), pp. 255-274.

[22] Ch. Herrmann, Alan Day's work on modular and Arguesian lattices, Algebra Universalis, Vol. 34, No. 3 (1995), pp. 35-60.

[23] D. Hobby and R. McKenzie, The Structure of Finite Algebras, volume 76 of Contemporary Mathematics, American Mathematical Society, 2000 .

[24] J. Järvinen and S. Radeleczki, Rough sets determined by tolerances, International Journal of Approximate Reasoning, Vol. 55, No. 6 (2014), pp. 1419-1438.

[25] B. Jónsson, Algebras whose congruence lattices are distributive, Mathematica Scandinavia, Vol. 21, No. 1 (1967), pp. 110-121.

[26] J. F. Peters and P. Wasilewski, Tolerance spaces: Origins, theoretical aspects and applications, Information Sciences: Informatics and Computer Science, Intelligent Systems, Applications, Vol. 195 (2012), pp. 211-225.

[27] J. Pogonowski, Tolerance spaces with applications to linguistics, Wydawnictwo Naukowe UAM, Poznań, 1983. 
[28] J. A. Szrejder, Równość, podobieństwo, porządek, Wydawnictwa Naukowo-Techniczne, Warszawa, 1975.

[29] E. Ch. Zeeman, The topology of the brain and visual perception, [in:] M. K. Fort (ed.), Topology of 3-Manifolds and related topics, New Jersey, 1962.

Jan Długosz University in Częstochowa

Institute of Philosophy

e-mail: j.grygiel@ujd.edu.pl 